\title{
Inhibition of microRNA-29c protects the brain in a rat model of prolonged hypothermic circulatory arrest
}

\author{
Yongchao Wang, MD, Tianxiang Gu, MD, PhD, Enyi Shi, MD, PhD, Lei Yu, MD, Chun Wang, MD, PhD,
} Yuhai Zhang, MD, PhD, and Qin Fang, MD

\section{ABSTRACT}

Objective: We sought to investigate the cerebroprotection of a novel microRNA mechanism by targeting peroxisome proliferator-activated receptor gamma coactivator 1-alpha in a rat model of prolonged deep hypothermia circulatory arrest.

Methods: The right carotid artery and jugular vein of male Sprague-Dawley rats were cannulated for cardiopulmonary bypass. Circulatory arrest was conducted for 60 minutes when the pericranial temperature was cooled to $18^{\circ} \mathrm{C}$. The sham group received the surgical procedure without cardiopulmonary bypass and deep hypothermia circulatory arrest; the deep hypothermia circulatory arrest group received cardiopulmonary bypass and deep hypothermia circulatory arrest; lentivirus control vector or lentiviral vector containing antagomiR-29c was given to the deep hypothermia circulatory arrest + vector group or the deep hypothermia circulatory arrest + antagomiR-29c group by intracerebroventricular administration 5 days before cardiopulmonary bypass $(n=8$, for each of the 4 groups $)$. Neurologic function was evaluated by the modified hole board test and beam balance task during 14 postoperative days. Expressions of caspase-3, peroxisome proliferator-activated receptor gamma coactivator 1-alpha, and miR-29c in the hippocampus were measured by Western blot and quantitative reverse transcription polymerase chain reaction. Malondialdehyde was measured using the Malondialdehyde Assay Kit (Beyotime, Jiangsu, China).

Results: Pretreatment with antagomiR-29c significantly decreased the expression of microRNA-29c and increased the expression of peroxisome proliferatoractivated receptor gamma coactivator 1 -alpha in the hippocampus $(P<.05$ vs deep hypothermia circulatory arrest group). The level of malondialdehyde in the hippocampus was lower in the deep hypothermia circulatory arrest + antagomiR-29c group $(P<.05$ vs deep hypothermia circulatory arrest group). The neurologic functions were markedly protected in rats pretreated with antagomiR-29c as evidenced by improvement of vestibulomotor and cognitive performance during the early postoperative period. In the deep hypothermia circulatory arrest + antagomiR-29c group, histologic scores of the hippocampus were improved and the level of caspase- 3 in the hippocampus was lower $(P<.05$ vs deep hypothermia circulatory arrest group).

Conclusions: Inhibition of miR-29c attenuates neurologic injuries induced by prolonged deep hypothermia circulatory arrest through a peroxisome proliferatoractivated receptor gamma coactivator 1-alpha pathway. (J Thorac Cardiovasc Surg 2015;150:675-84)

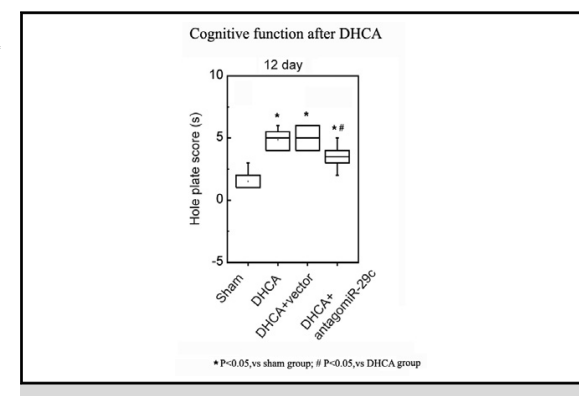

Inhibition of miR-29c attenuates neurologic dysfunction after DHCA.

\section{Central Message}

Inhibition of miR-29c by intracerebroventricular administration of lentivirus vectors containing antagomiR-29c attenuates neurologic injuries induced by prolonged DHCA through a PGC- $1 \alpha$ pathway.

\section{Perspective}

The current study highlights the potential role of miRNAs in neuroprotection after DHCA, a special procedure of brain ischemia. The results indicate that inhibition of miR-29c conducts neuroprotective effects against injuries induced by DHCA through a PGC- $1 \alpha$-dependent pathway. To our best knowledge, this is the first report that miRNAs play a critical role in neurologic injuries of DHCA. Regulation of miRNAs may become a novel therapeutic strategy for attenuation of neurologic injuries after DHCA.

See Editorial Commentary page 684.
From the Department of Cardiac Surgery, First Affiliated Hospital, China Medical University, Shenyang, China.

This work was supported by Grant No. 81171175 from the National Natural Science Foundation of China, Beijing, P. R. China.

Read at the 95th Annual Meeting of The American Association for Thoracic Surgery, Seattle, Washington, April 25-29, 2015.

Received for publication Jan 9, 2015; revisions received March 26, 2015; accepted for publication April 2, 2015; available ahead of print Aug 5, 2015.
Address for reprints: Tianxiang Gu, MD, PhD, Department of Cardiac Surgery, Firs Affiliated Hospital, China Medical University, Shenyang, China 110001 (E-mail: cmugtx@sina.com).

$0022-5223 / \$ 36.00$

Copyright (C) 2015 by The American Association for Thoracic Surgery http://dx.doi.org/10.1016/j.jtcvs.2015.04.062 


$$
\begin{aligned}
\text { Abbreviations and Acronyms } \\
\text { CPB }=\text { cardiopulmonary bypass } \\
\text { DHCA }=\text { deep hypothermia circulatory arrest } \\
\text { MDA }=\text { malondialdehyde } \\
\text { miR-29c }=\text { microRNA-29c } \\
\text { miRNA }=\text { microRNA } \\
\text { PGC-1 } 1 \alpha=\text { peroxisome proliferator-activated } \\
\text { receptor gamma coactivator } 1 \text {-alpha } \\
\text { ROS }=\text { reactive oxygen species }
\end{aligned}
$$

Supplemental material is available online.

Cardiopulmonary bypass (CPB) and deep hypothermia circulatory arrest (DHCA) are required for the repair of complex pediatric and adult congenital cardiac and aortic arch lesions. Although the overall mortality and morbidity after surgery with DHCA have been substantially improved, neurologic deficit induced by DHCA remains a major complication. New onset of cerebrovascular events was seen in $5.4 \%$ of patients after noncomplex hemiarch surgery with the aid of DHCA. ${ }^{1}$ Moreover, it was also reported that approximately $25 \%$ patients had a temporary neurologic dysfunction after $\mathrm{DHCA},{ }^{2}$ and that the incidence of neuropsychologic deficit within 12 weeks after the operation with DHCA was as high as $55 \%{ }^{3}$

Ischemia-reperfusion injury caused by circulation arrest contributes mainly to the neurologic injury after DHCA. It has been reported that one of the principal roots for ischemia-reperfusion injury is the oxidative stress trigged by the excessive production of free radicals. ${ }^{4}$ Overproduction of reactive oxygen species (ROS) as by-products generated from mitochondria has been implicated in acute brain injuries, such as stroke from cerebral ischemia. ${ }^{5}$ Mitochondria activate signaling pathways through ROS production and regulate mitochondria-dependent apoptosis pathways after cerebral ischemia. ${ }^{6}$ Oxidative stress and apoptotic neuronal death signaling pathways also have been involved in the brain in patients undergoing CPB and DHCA. ${ }^{7}$ Peroxisome proliferator-activated receptor gamma coactivator 1alpha (PGC- $1 \alpha$ ) is a potent stimulator of mitochondrial biogenesis and respiration, and powerfully regulates oxidative and mitochondrial metabolism in a number of tissues, including the central nerve system. ${ }^{8}$ However, it is still not known whether PGC- $1 \alpha$ is expressed in the brain after DHCA and functions as a regulator of oxidative stress and apoptotic neuronal death.

As negative regulators of gene expression, ${ }^{9}$ microRNAs (miRNAs) have been identified to play critical roles in brain ischemia and injury, neuroprotection, and neurodegeneration. ${ }^{10}$ Transient focal ischemia induces extensive temporal changes in rat cerebral microRNAome. ${ }^{11}$ Collective evidence shows that miR-29c is enriched in the brain ${ }^{12}$ and plays dramatic roles in the brain development ${ }^{13}$ and neurite outgrowth ${ }^{14}$ With the help of bioinformatics-based databases ${ }^{15,16}$ and the published report, ${ }^{17}$ miR-29c is indicated to be an endogenous regulator of PGC- $1 \alpha$. Therefore, we tried to explore the possible neuroprotection of using microRNA-29c (miRNA-29c) as a regulator of PGC- $1 \alpha$ in the brain after DHCA in the current study.

\section{MATERIALS AND METHODS \\ Animal Care}

Male Sprague-Dawley rats weighing approximately $350 \mathrm{~g}$ were used in the current study. The experimental protocol was approved by the Ethics Review Committee for Animal Experimentation of China Medical University (Shenyang, China) and was in accordance with the Guide for the Use and Care of Laboratory Animals (National Institutes of Health, Bethesda, Md).

\section{Cardiopulmonary Bypass and Deep Hypothermia Circulatory Arrest}

A rat model of $\mathrm{CPB}$ and DHCA was conducted according to the method reported previously. ${ }^{18,19}$ Briefly, animals were anesthetized with chloral hydrate and isoflurane in $60 \%$ oxygen, and the anesthesia was maintained with $2.0 \%$ to $2.5 \%$ isoflurane and fentanyl $(25 \mathrm{mg} / \mathrm{kg}$, intravenous, as a bolus injection). After orotracheal intubation with a 14G cannula, the animals were mechanically ventilated with a tidal volume of $10 \mathrm{~mL} \cdot \mathrm{kg}^{-1}$ and respiratory rate of 60 to 65 beats $\cdot \mathrm{min}^{-1}$. After a systemic heparinization (150 IU), the right carotid artery was cannulated for aortic inflow. A multistaged venous return cannula was placed near the junction of the inferior vena cava and right atrium through the right external jugular vein, and the tail artery was cannulated for blood pressure monitoring and blood sampling. The CPB circuit consisted of a venous reservoir, a peristaltic pump, and a custom-designed small-volume oxygenator. The entire circuit was primed with $10 \mathrm{~mL}$ of $6 \%$ hetastarch. Physiologic data, including mean artery pressure, temperature, and blood gas analysis, were collected. CPB was conducted at a flow rate of 160 to $180 \mathrm{~mL} \cdot \mathrm{kg}^{-1} \cdot \mathrm{min}^{-1}$ and was consecutively decreased by half during the cooling period. Rats were cooled to a pericranial temperature of $18^{\circ} \mathrm{C}$ over 30 minutes ( $\mathrm{pH}$ stat cooling was used) by using a heat exchanger and topical cooling with ice bags and a cooling blanket. Then CPB was stopped, and venous blood was drained to the reservoir carefully. DHCA was confirmed by the absence of systole and measurable blood pressure. After 60 minutes of DHCA, CPB was reinstituted and the flow rate was gradually increased to 160 to $180 \mathrm{~mL} \cdot \mathrm{kg}^{-1} \cdot \mathrm{min}^{-1}$. The rats were rewarmed to a pericranial temperature of $34^{\circ} \mathrm{C}$ over 30 minutes and then CPB was weaned. After decannulation, rats were maintained anesthetized with $0.5 \%$ to $1.2 \%$ isoflurane, intubated, and ventilated for 2 hours while warming the rats to a pericranial temperature of $36^{\circ} \mathrm{C}$ to $37^{\circ} \mathrm{C}$. After spontaneous ventilation had resumed, animals were extubated and allowed to recover in an oxygenenriched environment for 12 hours. Animals demonstrating signs of severe neurologic dysfunction, such as immediate signs of cerebral death, were excluded from the protocol.

\section{Lentivirus Vectors for AntagomiR-29c}

Chemical modified antisense oligonucleotides of rat miR-29c (antagomiR-29c) lentivirus gene transfer vector were constructed by Genechem (Shanghai, China). The recombinant lentivirus vector of antagomiR-29c 


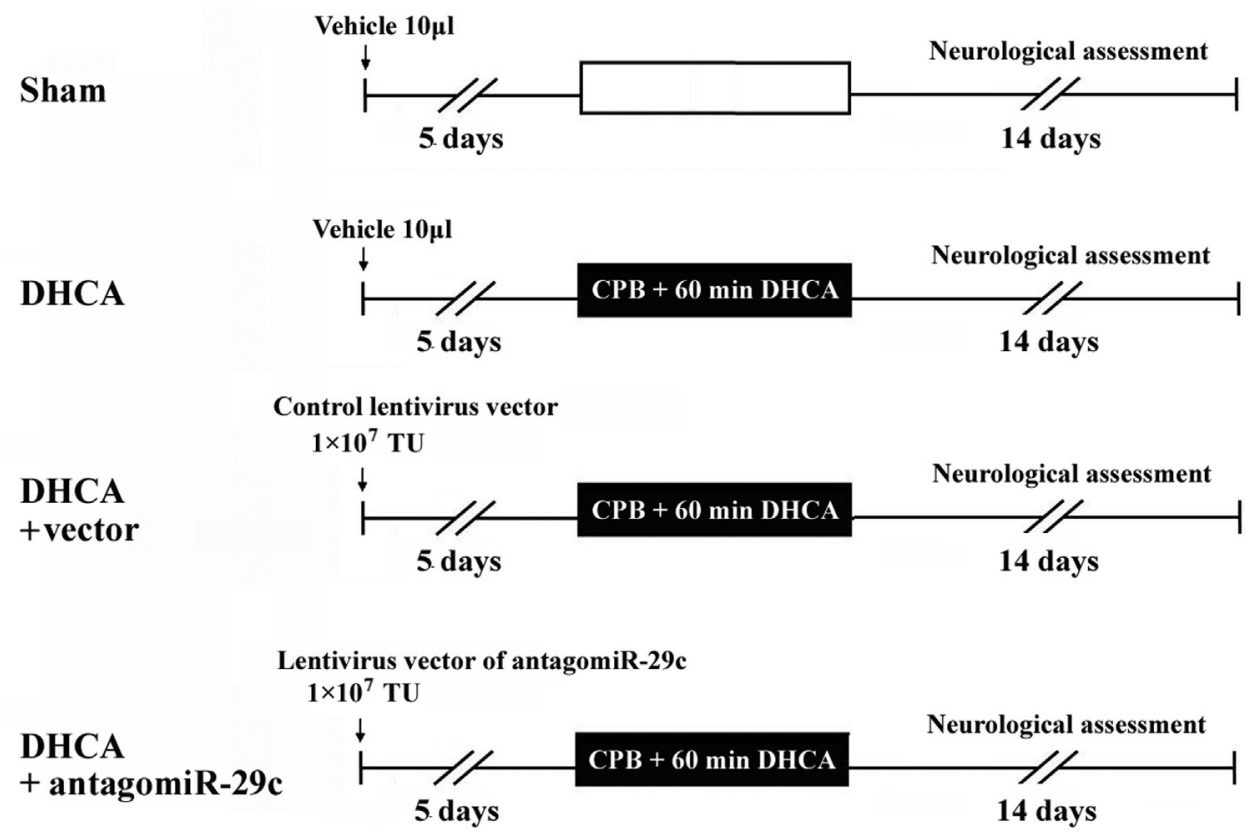

FIGURE 1. Experimental groups and protocol. $C P B$, Cardiopulmonary bypass; $D H C A$, deep hypothermic circulatory arrest; $T U$, transfection unit.

and the control lentivirus vector without antagomiR-29c were prepared and titered to $1 \times 10^{9}$ transfection unit $\cdot \mathrm{mL}^{-1}$.

\section{Intracerebroventricular Injection}

After anesthesia, a puncture needle was implanted into the lateral ventricle (bregma; $0.8 \mathrm{~mm}$ posterior, $-4.8 \mathrm{~mm}$ dorsoventral, $-1.5 \mathrm{~mm}$ lateral). ${ }^{20}$ Proper placement of the needle in the lateral ventricle was indicated by the presence of cerebral spinal fluid in the needle hub. ${ }^{11}$ Once correct needle placement was confirmed, the solutions were injected over 1 minute. The animals were included in the study only if they had a normal neurologic function 3 days after the intracerebroventricular injection.

\section{Experimental Protocol}

Rats were randomly assigned to 4 groups, as shown in Figure 1. The sham group received only the surgical procedure without $\mathrm{CPB}$ and DHCA. The other 3 groups were subjected to CPB and a 60-minute DHCA. The DHCA + antagomiR-29c group and the DHCA + vector group received intracerebroventricular injection of approximately $1 \times 10^{7}$ transfection unit lentivirus vectors of antagomiR-29c or the control lentivirus vectors in a total fluid volume of $10 \mu \mathrm{L} 5$ days before the surgical procedure, whereas the vehicle alone of the same volume was given to the sham group and DHCA group.

In a parallel series of experiments, rat brains were collected 2 hours after the surgical procedure from the 4 groups ( $n=4$ per group) for evaluating the expressions of miR-29c, PGC- $1 \alpha$, and caspase- 3 and measuring malondialdehyde (MDA) levels.

\section{Neurologic Assessment}

Cognitive functions were assessed by the modified hole board test as reported previously. ${ }^{18}$ Briefly, rats were housed in an area divided into a home cage $(80 \times 60 \times 50 \mathrm{~cm})$ and a test arena $(40 \times 60 \times 50 \mathrm{~cm})$ with a centrally placed hole board $(20 \times 40 \mathrm{~cm})$. Fifteen holes covered by lids were staggered on the board. After being opened, coil springs forced the lids back to their original position. Three holes baited with puffed rice were marked with white tape. All holes were flavored with the aroma of black currants to cover the odor and smell of the puffed rice. The scores for the modified hole board test with a range from 0 (normal) to 24 (severe cognitive dysfunction) are provided in Table E1. Vestibulomotor function was assessed using a beam balance task where the rat was placed on a $1.5 \mathrm{~cm}$ wide beam. The duration it remained on the beam was recorded (maximum $=60$ seconds). ${ }^{21}$

\section{Malondialdehyde Measurement}

As a marker of oxidative stress and free oxygen radical-mediated damage, MDA levels of the hippocampus were measured using the MDA Assay Kit (Beyotime, Jiangsu, China) according to the procedure recommended by the manufacturer. MDA concentration was calculated in nanomoles per gram of protein.

\section{Histologic Study}

After evaluation of the vestibulomotor and cognitive function during a 14-day postoperative period, animals were killed for histologic evaluation. Brain tissues were sectioned $(5 \mu \mathrm{m})$ and stained with hematoxylin-eosin. The neuronal damage of the hippocampus CA1 region was evaluated qualitatively by a pathologist blinded to the grouping. Neurons showing features such as shrunken cell bodies, triangulated, pyknotic nuclei, and eosinophilic cytoplasm were identified as a dead neuron. Normal neurons and death neurons were counted using a defined rectangular field area with magnification of $200 \times$. A pathologic score was used to evaluate the neurologic damage: $0=$ no damage; $1=0 \%$ to $12.5 \%$ damage; $2=12.5 \%$ to $25 \%$ damage; $3=25 \%$ to $50 \%$ damage; and $4=$ more than $50 \%$ damage.

\section{microRNA Extraction and Quantitative Reverse Transcription Polymerase Chain Reaction}

Total RNA was isolated by using Trizol reagent (Invitrogen, Carlsbad, Calif) according to the manufacturer's instruction. The first-strand cDNA was generated using the Reverse Transcription System Kit (Invitrogen). Real-time polymerase chain reaction was performed according to a standard protocol using a StepOne Plus system (Applied Biosystems, Foster 
TABLE 1. Physiologic data

\begin{tabular}{|c|c|c|c|c|c|c|c|c|}
\hline & \multirow[b]{2}{*}{ Group } & \multirow[b]{2}{*}{ Baseline } & \multicolumn{2}{|c|}{ CPB } & \multicolumn{2}{|c|}{ Post-CPB } & \multirow[b]{2}{*}{$\mathbf{F}$} & \multirow[b]{2}{*}{$P$ value } \\
\hline & & & $\begin{array}{l}\text { Cooling } \\
20 \text { min }\end{array}$ & $\begin{array}{l}\text { Warming } \\
20 \text { min }\end{array}$ & $15 \mathrm{~min}$ & $120 \mathrm{~min}$ & & \\
\hline \multirow[t]{4}{*}{ Weight (g) } & Sham & $351 \pm 14$ & & & & & $\mathrm{~F}=0.172$ & .914 \\
\hline & DHCA & $350 \pm 10$ & & & & & & \\
\hline & DHCA + vector & $353 \pm 12$ & & & & & & \\
\hline & DHCA + antagomir-29c & $354 \pm 8$ & & & & & & \\
\hline \multirow[t]{4}{*}{ MAP (mm Hg) } & Sham & $74 \pm 4$ & & & & & & \\
\hline & DHCA & $75 \pm 5$ & $45 \pm 4^{*}$ & $49 \pm 3^{*}$ & $75 \pm 4$ & $76 \pm 6$ & $\mathrm{~F}($ group $)=1.665$ & .194 \\
\hline & DHCA + vector & $76 \pm 7$ & $46 \pm 3^{*}$ & $49 \pm 4^{*}$ & $74 \pm 4$ & $75 \pm 4$ & $\mathrm{~F}($ time $)=290.459$ & .000 \\
\hline & DHCA + antagomiR-29c & $79 \pm 7$ & $46 \pm 4^{*}$ & $50 \pm 3^{*}$ & $77 \pm 5$ & $76 \pm 6$ & & \\
\hline \multirow[t]{4}{*}{ Heart rate } & Sham & $317 \pm 11$ & & & & & & \\
\hline & DHCA & $321 \pm 10$ & $86 \pm 5^{*}$ & $303 \pm 12 *$ & $315 \pm 5$ & $321 \pm 10$ & $\mathrm{~F}($ group $)=0.077$ & .926 \\
\hline & DHCA + vector & $313 \pm 15$ & $91 \pm 9^{*}$ & $301 \pm 12 *$ & $318 \pm 10$ & $320 \pm 8$ & $\mathrm{~F}($ time $)=1983.430$ & .000 \\
\hline & DHCA + antagomiR-29c & $318 \pm 17$ & $88 \pm 8^{*}$ & $310 \pm 13^{*}$ & $316 \pm 14$ & $318 \pm 10$ & & \\
\hline \multirow{4}{*}{$\begin{array}{l}\text { Pericranial } \\
\quad \text { temperature }\left({ }^{\circ} \mathrm{C}\right)\end{array}$} & Sham & $33.6 \pm 0.2$ & & & & & & \\
\hline & DHCA & $33.7 \pm 0.2$ & $21.6 \pm 0.5^{*}$ & $28.3 \pm 0.7^{*}$ & $35.1 \pm 0.2^{*}$ & $35.7 \pm 0.4^{*}$ & $\mathrm{~F}($ group $)=0.199$ & .820 \\
\hline & $\mathrm{DHCA}+$ vector & $33.8 \pm 0.2$ & $21.8 \pm 0.6^{*}$ & $27.9 \pm 1.0^{*}$ & $35.2 \pm 0.4^{*}$ & $35.8 \pm 0.3^{*}$ & $\mathrm{~F}($ time $)=3082.746$ & .000 \\
\hline & DHCA + antagomiR-29c & $33.8 \pm 0.2$ & $21.2 \pm 0.5^{*}$ & $28.1 \pm 1.1^{*}$ & $35.2 \pm 0.3^{*}$ & $35.8 \pm 0.2^{*}$ & & \\
\hline \multirow[t]{4}{*}{$\mathrm{pH}$} & Sham & $7.4 \pm 0.02$ & & & & & & \\
\hline & DHCA & $7.41 \pm 0.02$ & $7.33 \pm 0.03^{*}$ & $7.45 \pm 0.02 *$ & $7.4 \pm 0.03$ & $7.44 \pm 0.03^{*}$ & $\mathrm{~F}($ group $)=1.898$ & .155 \\
\hline & DHCA + vector & $7.4 \pm 0.02$ & $7.32 \pm 0.03^{*}$ & $7.46 \pm 0.02^{*}$ & $7.38 \pm 0.05$ & $7.43 \pm 0.03 *$ & $\mathrm{~F}(\mathrm{time})=59.717$ & .000 \\
\hline & DHCA + antagomiR-29c & $7.42 \pm 0.03$ & $7.35 \pm 0.04 *$ & $7.46 \pm 0.02^{*}$ & $7.39 \pm 0.03$ & $7.45 \pm 0.02 *$ & & \\
\hline \multirow[t]{4}{*}{$\mathrm{PaO}_{2}(\mathrm{~mm} \mathrm{Hg})$} & Sham & $262 \pm 51$ & & & & & & \\
\hline & DHCA & $258 \pm 57$ & $417 \pm 60^{*}$ & $317 \pm 61 *$ & $128 \pm 18^{*}$ & $306 \pm 48^{*}$ & $\mathrm{~F}($ group $)=2.660$ & .074 \\
\hline & DHCA + vector & $271 \pm 80$ & $429 \pm 77 *$ & $323 \pm 79 *$ & $138 \pm 27 *$ & $360 \pm 61 *$ & $\mathrm{~F}($ time $)=73.082$ & .000 \\
\hline & DHCA + antagomiR-29c & $274 \pm 73$ & $457 \pm 85^{*}$ & $342 \pm 78 *$ & $142 \pm 12^{*}$ & $373 \pm 88^{*}$ & & \\
\hline \multirow[t]{4}{*}{$\mathrm{PACO}_{2}(\mathrm{~mm} \mathrm{Hg})$} & Sham & $42 \pm 2$ & & & & & & \\
\hline & DHCA & $42 \pm 3$ & $36 \pm 2 *$ & $38 \pm 3^{*}$ & $44 \pm 3 *$ & $41 \pm 4$ & $\mathrm{~F}($ group $)=0.127$ & .881 \\
\hline & DHCA + vector & $40 \pm 5$ & $37 \pm 4^{*}$ & $39 \pm 4 *$ & $45 \pm 2 *$ & $41 \pm 5$ & $\mathrm{~F}($ time $)=18.964$ & .000 \\
\hline & DHCA + antagomiR-29c & $40 \pm 4$ & $37 \pm 3^{*}$ & $38 \pm 3^{*}$ & $44 \pm 3 *$ & $41 \pm 2$ & & \\
\hline
\end{tabular}

$\mathrm{CPB}$, Cardiopulmonary bypass; $\mathrm{DHCA}$, deep hypothermia circulatory arrest; $\mathrm{MAP}$, mean arterial pressure; $\mathrm{PACO}_{2}$, alveolar carbon dioxide tension; $\mathrm{PaO}_{2}$, arterial oxygen tension $* P<.05$ versus baseline of the same group. $\mathrm{n}=8$ per group.

City, Calif). The U6 served as an internal control. Changes in expression were determined by the $2^{-\Delta \Delta \mathrm{CT}}$ method.

\section{Western Blot}

Caspase- 3 and PGC- $1 \alpha$ were analyzed by sodium dodecyl sulfate polyacrylamide gel electrophoresis and immunoblotted with anti-caspase- 3 and anti-PGC- $1 \alpha$ antibody (Abcam, Cambridge, Mass), respectively. The caspase- 3 and PGC- $1 \alpha$ expressions in each brain tissue sample were analyzed with National Institutes of Health Image and quantified as a relative fold to the sham group after normalization with $\beta$-actin.

\section{Statistical Analysis}

Parametric values are reported as mean \pm standard deviation. The Kruskal-Wallis test was used for nonparametric values, and the Mann-Whitney $U$ test was used as a posttest to identify the specific differences among the groups. Parametric values were analyzed by 1-way or 2-way repeatedmeasures (time and group) analysis of variance followed by the Bonferroni post hoc test. Statistical analyses were performed using SPSS version 19.0 (SPSS Inc, Chicago, Ill).

\section{RESULTS}

\section{Mortality and Exclusion}

A total of 37 rats were enrolled in the protocol for neurologic assessment. One rat in the DHCA + vector group was excluded because of cerebral death after the surgical procedure, and 4 rats were excluded because of death during the recovery period ( 1 in sham group; 1 in DHCA + antagomiR-29c group; 2 in DHCA group). Complete data were obtained in the remaining 32 rats $(n=8$, for each of the 4 groups).

\section{Physiologic Parameters}

Table 1 indicates the physiologic parameters from the 4 groups during the surgical procedure. No significant differences in body weight and physiologic values of the baseline were observed among the 4 groups $(n=8$, for each of the 4 groups). The hemodynamic data and the data of blood gas analysis were similar at any time point during the CPB and DHCA among the 3 groups receiving $\mathrm{CPB}$ and DHCA $(P>.05)$.

\section{Neurologic Assessment}

Vestibulomotor function assessed by the beam balance task is summarized in Figure $2(n=8$, for each of the 4 

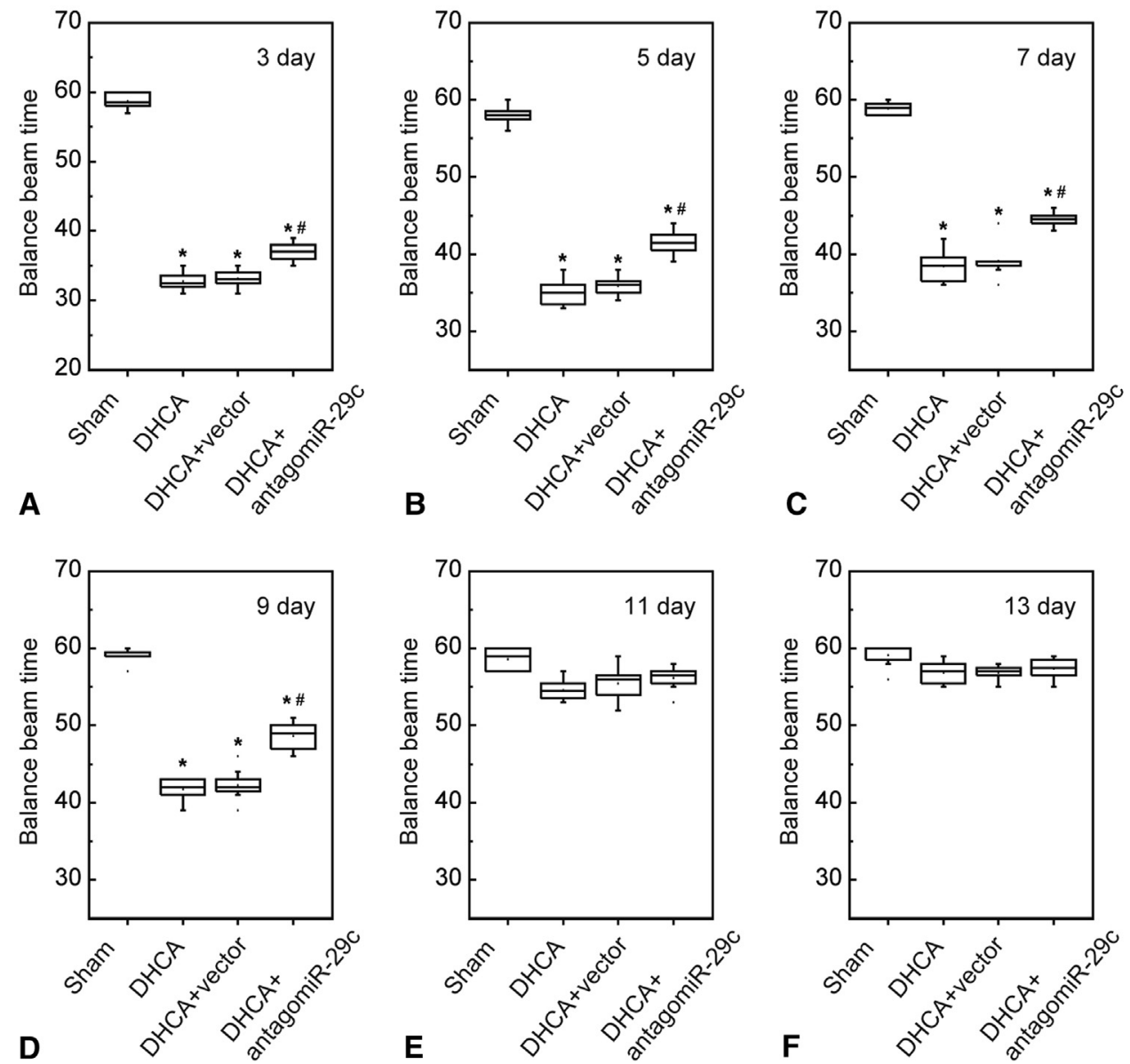

FIGURE 2. A-F, Vestibulomotor function assessed by the beam balance task after the surgical procedure. $\mathrm{n}=8$, for each of the 4 groups. *P<.01 versus sham group. $\# P<.05$ versus DHCA group. DHCA, Deep hypothermic circulatory arrest.

groups). Vestibulomotor functions of animals in the DHCA and DHCA + vector groups were damaged remarkably during the early period after DHCA, as evidenced by the shorter balance beam time compared with the sham group
$(P<.01$ at postoperative days $3,5,7$, and 9 of the DHCA group and DHCA + vector group), and such functions were recovered in approximately 2 weeks (vs sham group, $P>.05$ at postoperative days 11 and 13 of the DHCA group
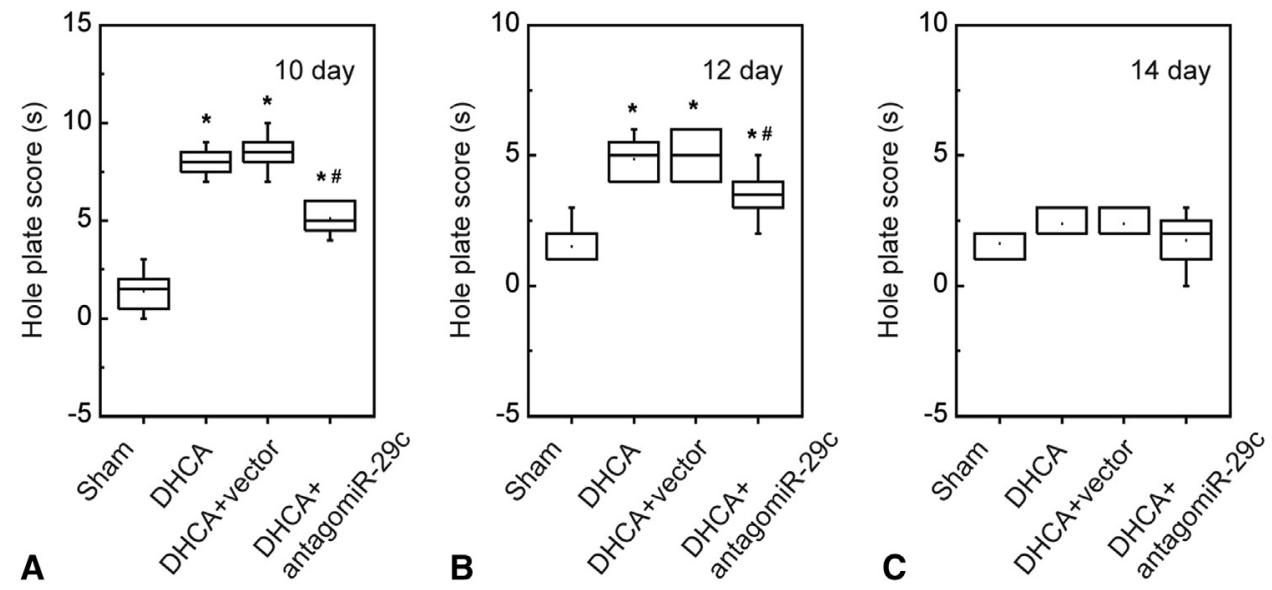

FIGURE 3. A-C, Cognitive function assessed by modified hole board test after the surgical procedure. $\mathrm{n}=8$, for each of the 4 groups. $* P<.05$ versus sham group. $\# P<.05$ versus DHCA group. DHCA, Deep hypothermic circulatory arrest. 


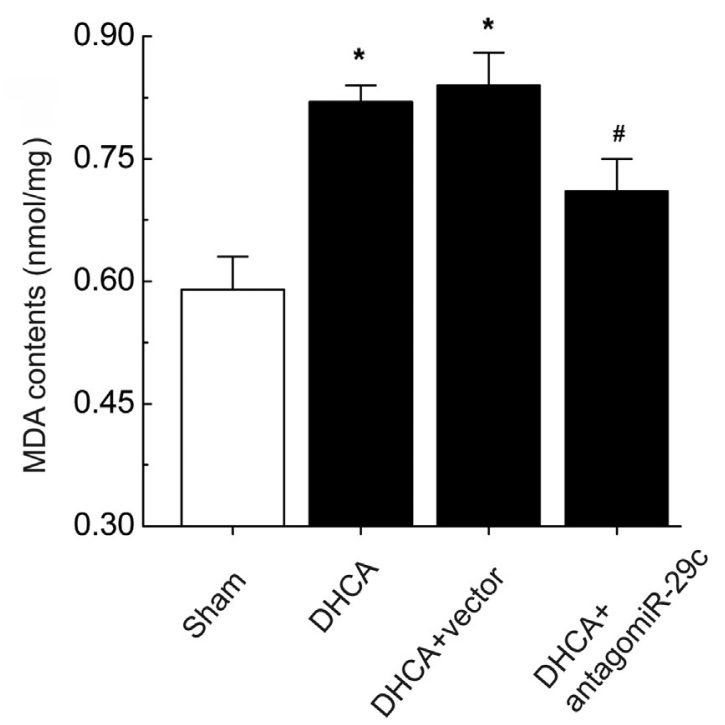

FIGURE 4. MDA contents in the hippocampus. $\mathrm{n}=4$, for each of the 4 groups. $* P<.05$ versus sham group. \#P<.05 versus DHCA group. $D H C A$, Deep hypothermic circulatory arrest; $M D A$, malondialdehyde.

and DHCA + vector group, respectively). Although damaged vestibulomotor functions were also detected in the rats with pretreatment with anatagomiR-29c during the early period after DHCA (vs sham group, $P<.01$ at postoperative days $3,5,7$, and 9 ), the vestibulomotor function of the DHCA + antagomiR-29c group was still protected to a significant extent when compared with the DHCA group $(P<.05$ at postoperative days 3, 5, 7, and 9$)$.

DHCA also affected the cognitive function of animals as illustrated in Figure 3. On postoperative days 10 and 12, the

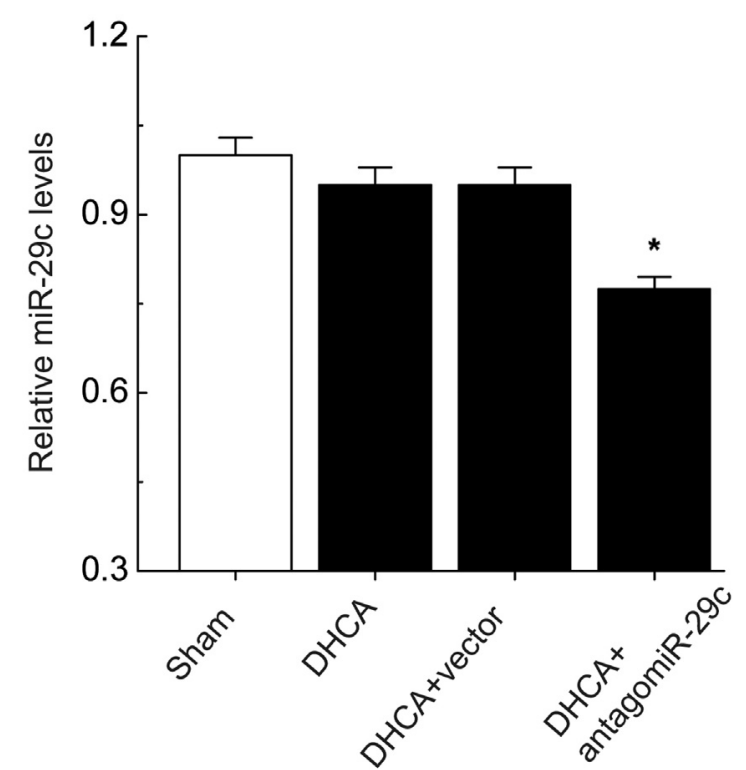

FIGURE 5. miR-29c expression in the hippocampus. $\mathrm{n}=4$, for each of the 4 groups. $* P<.05$ versus DHCA group. $D H C A$, Deep hypothermic circulatory arrest; $m i R-29 c$, microRNA-29c. hole plate scores of the DHCA, DHCA + vector, and DHCA + antagomiR-29c groups were higher than in the sham group $(P<.05)$. However, the plate scores of the DHCA + antagomiR-29c group at postoperative days 10 and 12 were still significantly lower than those in the DHCA group $(P<.05)$. On postoperative day 14 , no significant differences of the plate scores were found among the 4 groups $(P=.064)$.

\section{Malondialdehyde Levels}

MDA levels were significantly increased in the DHCA and DHCA + vector groups compared with the sham group $(P<.05)$ (Figure 4). Intracerebroventricular injection of antagomiR-29c remarkably reduced the level of MDA in the DHCA + antagomiR-29C group $(P<.05$ vs DHCA group).

\section{Expression of microRNA-29c}

The results of quantitative reverse transcription polymerase chain reaction showed that miR-29c was enriched in the hippocampus (Figure 5). DHCA slightly decreased the expression of miR-29c in the DHCA and DHCA + vector groups ( $P>.05$ vs sham group). However, intracerebroventricular injection of antagomiR-29c before DHCA significantly decreased the expression of miR-29c $(P<.05$ vs DHCA group).

\section{Western Blot}

As illustrated in Figure 6, $A$ and $C$, PGC- $1 \alpha$ was expressed in the hippocampus of rats. After DHCA, PGC$1 \alpha$ expressions decreased significantly in the DHCA and DHCA + vector groups $(P<.01$ vs sham group). Transfection with lentivirus vectors of antagomiR-29c markedly increased the expression of PGC- $1 \alpha$ in the DHCA + antagomiR-29c group ( $P<.01$ vs DHCA group).

Densitometric analysis of the Western blot images revealed a weak signal of caspase- 3 in the sham group (Figure 6, $B$ and $D$ ). The expression of caspase-3 increased dramatically after DHCA in the DHCA and DHCA + vector groups $(P<.01$ vs sham group), whereas pretreatment with antagomiR-29c significantly reduced the expression of caspase-3 in the DHCA + antagomiR29 c group $(P<.01$ vs DHCA group).

\section{Histologic Examination}

Representative coronal brain sections of the hippocampus and the cerebellar vermis stained with hematoxylineosin are shown in Figure 7, A. In sham animals, neurons were intact with a clear shape and abundant cytoplasm. In the DHCA and DHCA + vector groups, many neurons with features of dead neuron (eg, shrunken cell bodies, triangulated, pyknotic nuclei, and eosinophilic cytoplasm) were detected. Pretreatment of antagomiR-29c attenuated the morphologic changes in the DHCA + antagomiR-29c 

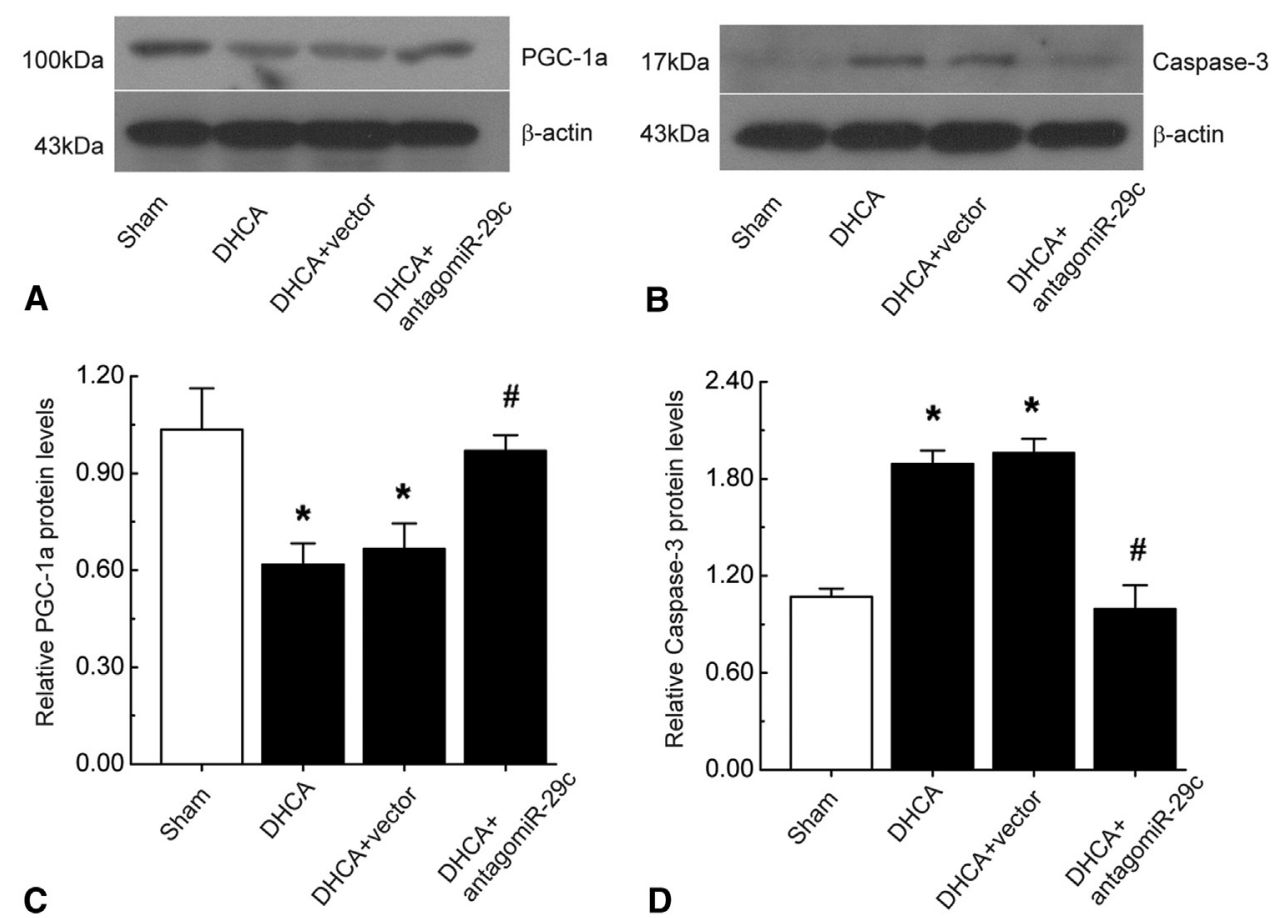

FIGURE 6. Results of Western blot. A and B, Representative Western blot pictures showing PGC- $1 \alpha$ or caspase- 3 expressions. C and D, Densitometric quantification of PGC- $1 \alpha$ or caspase- 3 expressions (folds to the sham group). $\mathrm{n}=4$, for each of the 4 groups. $* P<.01$ versus sham group. \#P<.01 versus DHCA group. DHCA, Deep hypothermic circulatory arrest; $P G C$ - $1 \alpha$, peroxisome proliferator-activated receptor gamma coactivator 1-alpha.

group, and most neurons were kept intact. The pathologic score of the DHCA + antagomiR-29c group was lower than that of the DHCA or DHCA + vector group $(P<.05)$ (Figure 7, $B$ ).

\section{DISCUSSION}

In the current study, $\mathrm{CPB}$ and DHCA were imitated in a long-term recovery model of rats, and the DHCA time was extended to 60 minutes beyond the duration of what is commonly required in the clinical practice. After DHCA, the level of MDA and the expression of caspase-3 in the hippocampus increased significantly, which indicated that ROS and mitochondria-dependent apoptosis pathways contributed to the neurologic deficits induced by DHCA. Antioxidant interventions have become the therapeutic strategy to reduce the cerebral damage related to both ischemia and reperfusion. ${ }^{22}$ One of the main findings of the current study is that inhibition of miR-29c, a brainenriched miRNA, reduced the level of MDA and apoptosis of the hippocampus after DHCA.

DHCA is an extreme variant of cerebral hypoperfusion, and the ischemia-reperfusion injury is still the principal root for the neurologic injury after DHCA. Neuron death and apoptosis are the key cellular events in the ischemic central nervous system. It is well established that multiple genes and their target proteins are responsible for such pathologic processes after ischemia-reperfusion. miRNAs recently have become a novel target for cerebral protection against ischemia. The dramatic roles of miRNAs, such as miR-134, miR-30a, and miR-592, in the pathophysiologic process of cerebral ischemia have been identified in different models. ${ }^{23-25}$ In the current study, inhibition of miRNA-29c demonstrated significant neuroprotection against neurologic injuries caused by a prolonged DHCA in rats. miR-29c is enriched in the brain and is activated as an endogenous regulator of BACE1 protein. ${ }^{12}$ miR-29c is expressed during mice brain development and increases neurite outgrowth by decreasing PTEN expression. ${ }^{14}$ Overexpression of miRNA-29a, also a member of miRNA-29 family, has been indicated to attenuate the neurologic injury after forebrain ischemia. ${ }^{26}$ However, the current data showed that miRNA-29c was a negative mediator of cerebral protection against neurologic injuries of DHCA.

Another major finding of the present study is that the expression of PGC- $1 \alpha$ in the hippocampus was markedly enhanced by inhibition of miRNA-29c. Activation of miR-29a-c in mouse livers consistently decreases protein levels of PGC-1a. ${ }^{17}$ PGC- $1 \alpha$ is highly expressed in tissues with high metabolic demands and is generally thought to be the master regulator of mitochondrial biogenesis and ROS scavenging enzymes, including manganese superoxide dismutase 2 and the uncoupling protein $2 .{ }^{27}$ PGC- $1 \alpha$ gene expression is induced in cultured skeletal myotubes by ischemia-like conditions. ${ }^{28}$ Hypoxia stimulates PGC- $1 \alpha$ 

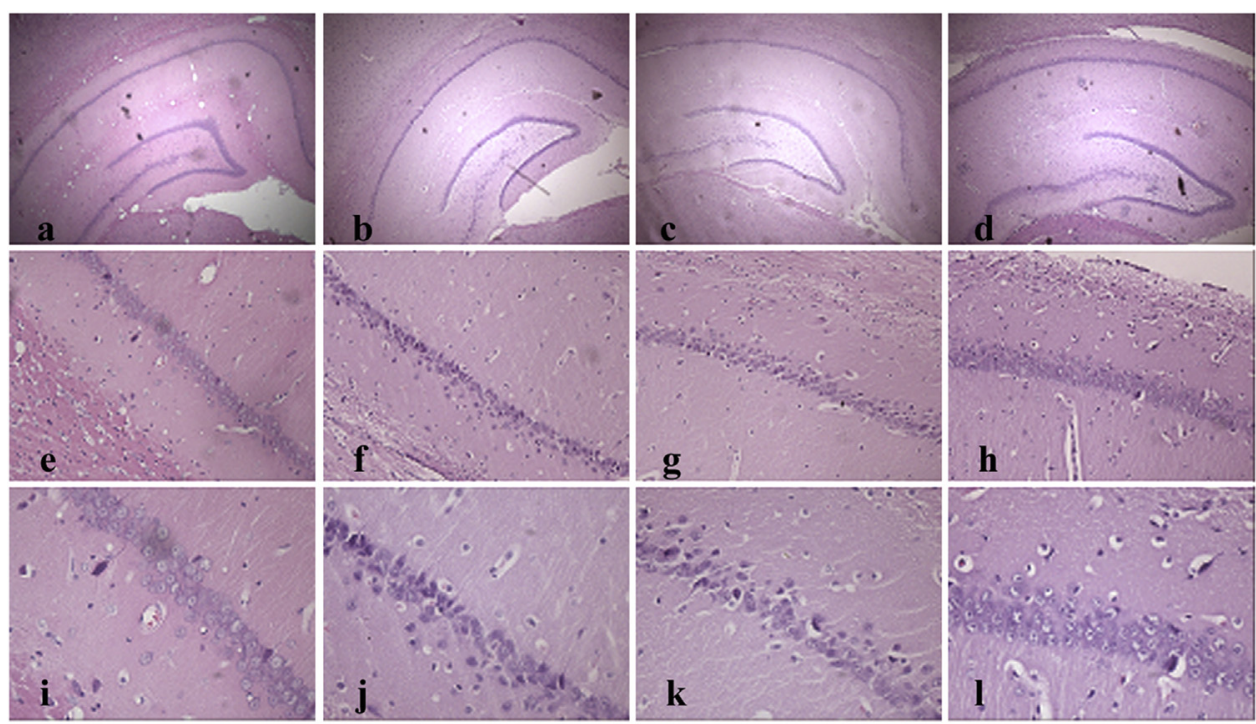

A

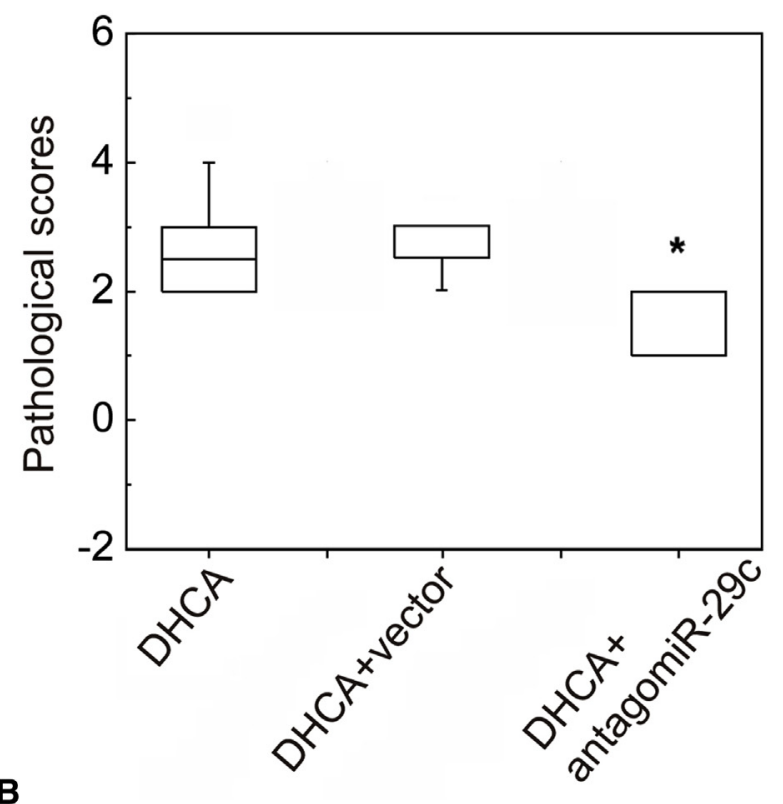

FIGURE 7. Histologic examination. A, Representative coronal brain sections of the hippocampus and cerebellar vermis stained with hematoxylin-eosin; a, b, c, d, magnification of 40×; e, f, g, h, magnification of 200×; i, j, k, l, magnification of 400×; a, e, i, sham group; b, f, j, DHCA group; c, g, k, DHCA + vector group; d, h, i, DHCA + antagomiR-29c group. B, Pathologic scores. $\mathrm{n}=8$, for each of the 4 groups. $* P<.05$ versus DHCA group. DHCA, Deep hypothermic circulatory arrest.

protein expression in the mouse cerebral subcortex. ${ }^{29}$ PGC$1 \alpha$ is induced by cerebral ischemia and provides a neuroprotective effect against ischemic brain injury in the hippocampus by ameliorating oxidative stress. ${ }^{30}$ An increased PGC- $1 \alpha$ expression protects cultured neural cells from oxidative stress-mediated cell death. ${ }^{27}$ Icariin protects against brain ischemic injury by increasing SIRT1 and PGC$1 \alpha$ expression. ${ }^{31}$ As the necessary mediator, PGC- $1 \alpha$ also conducts protective effects against brain ischemia induced by alpha-lipoic acid. ${ }^{32}$ Collectively, PGC- $1 \alpha$ is vital for neuron survival under the condition of ischemia or hypoxia through a mitochondrial pathway. Therefore, it is plausible that PGC1- $\alpha$ is the target protein by which inhibition of miR-29c conducts neuroprotection after DHCA in rats.

\section{Study Limitations}

Because of the large number of possible targets, the mode of action for miR-29c regulating PGC- $1 \alpha$ is not well established in the current study, which needs to be investigated in further research. Another limitation of the current study is that other target proteins may be implicated in the neuroprotection of miR-29c and should be identified in detail. 


\section{CONCLUSIONS}

The current study highlights the potential role of miRNAs in neuroprotection after DHCA, a special procedure of brain ischemia. The results indicate that inhibition of miR-29c conducts powerful neuroprotective effects against injuries induced by DHCA through a PGC- $1 \alpha-$ dependent pathway. To the best of our knowledge, this is the first report that miRNAs play a critical role in neurologic injuries of DHCA. Regulation of miRNAs may be a novel therapeutic strategy for attenuation of neurologic injuries after DHCA.

\section{Conflict of Interest Statement}

Authors have nothing to disclose with regard to commercial support.

You can watch a Webcast of this AATS meeting presentation by going to: http://webcast.aats.org/2015/Video/ Tuesday/04-28-15_6B_0730_Gu.mp4

\section{References}

1. Kaneko T, Aranki SF, Neely RC, Yazdchi F, McGurk S, Leacche M, et al. Is there a need for adjunct cerebral protection in conjunction with deep hypothermic circulatory arrest during noncomplex hemiarch surgery? J Thorac Cardiovasc Surg. 2014; 148:2911-7.

2. Ergin MA, Uysal S, Reich DL, Apaydin A, Lansman SL, McCullough JN, et al. Temporary neurological dysfunction after deep hypothermic circulatory arrest: a clinical marker of long-term functional deficit. Ann Thorac Surg. 1999;67: $1887-90$

3. Harrington DK, Bonser M, Moss A, Heafield MT, Riddoch MJ, Bonser RS, et al. Neuropsychometric outcome following aortic arch surgery: a prospective randomized trial of retrograde cerebral perfusion. J Thorac Cardiovasc Surg. 2003;126:638-44

4. Carden DL, Granger DN. Pathophysiology of ischaemia reperfusion injury. J Pathol. 2000;190:255-66.

5. Chen SD, Yang DI, Lin TK, Shaw FZ, Liou CW, Chuang YC. Roles of oxidative stress, apoptosis, PGC- $1 \alpha$ and mitochondrial biogenesis in cerebral ischemia. Int J Mol Sci. 2011;12:7199-215.

6. Niizuma K, Yoshioka H, Chen H, Kim GS, Jung JE, Katsu M, et al. Mitochondrial and apoptotic neuronal death signaling pathways in cerebral ischemia. Biochim Biophys Acta. 2010;1802:92-9.

7. Shen L, Wang J, Liu K, Wang C, Wang C, Wu H, et al. Hydrogen-rich saline is cerebroprotective in a rat model of deep hypothermic circulatory arrest. Neurochem Res. 2011;36:1501-11.

8. Shoag J, Arany Z. Regulation of hypoxia-inducible genes by PGC-1 alpha. Arterioscler Thromb Vasc Biol. 2010;30:662-6.

9. Kim VN, Han J, Siomi MC. Biogenesis of small RNAs in animals. Nat Rev Mol Cell Biol. 2009;10:126-39.

10. Saugstad JA. MicroRNAs as effectors of brain function with roles in ischemia and injury, neuroprotection, and neurodegeneration. J Cereb Blood Flow Metab. 2010;30:1564-76

11. Dharap A, Bowen K, Place R, Li LC, Vemuganti R. Transient focal ischemia induces extensive temporal changes in rat cerebral microRNAome. J Cereb Blood Flow Metab. 2009;29:675-87.

12. Zong Y, Wang H, Dong W, Quan X, Zhu H, Xu Y, et al. miR-29c regulates BACE1 protein expression. Brain Res. 2011;1395:108-15.

13. Podolska A, Kaczkowski B, Kamp Busk P, Søkilde R, Litman T, Fredholm M, et al. MicroRNA expression profiling of the porcine developing brain. PLoS One. 2011;6:e14494.

14. Zou H, Ding Y, Shi W, Xu X, Gong A, Zhang Z, et al. MicroRNA-29c/PTEN pathway is involved in mice brain development and modulates neurite outgrowth in PC12 cells. Cell Mol Neurobiol. 2015;35:313-22.

15. Lewis BP, Shih IH, Jones-Rhoades MW, Bartel DP, Burge CB. Prediction of mammalian microRNA targets. Cell. 2003;115:787-98.

16. Friedman RC, Farh KK, Burge CB, Bartel DP. Most mammalian mRNAs are conserved targets of microRNAs. Genome Res. 2009;19:92-105.
17. Liang J, Liu C, Qiao A, Cui Y, Zhang H, Cui A, et al. MicroRNA-29a-c decrease fasting blood glucose levels by negatively regulating hepaticgluconeogenesis. $J$ Hepatol. 2013;58:535-42.

18. Jungwirth B, Mackensen GB, Blobner M, Neff F, Reichart B, Kochs EF, et al. Neurologic outcome after cardiopulmonary bypass with deep hypothermic circulatory arrest in rats: description of a new model. J Thorac Cardiovasc Surg. 2006; 131:805-12.

19. Shim JK, Ma Q, Zhang Z, Podgoreanu MV, Mackensen GB. Effect of pregabalin on cerebral outcome after cardiopulmonary bypass with deep hypothermic circulatory arrest in rats. J Thorac Cardiovasc Surg. 2014;148:298-303.

20. Paxinos G, Watson C. The Rat in Stereotaxic Coordinates. 4th ed. New York, NY Academic Press; 1998.

21. Drabek T, Fisk JA, Dixon CE, Garman RH, Stezoski J, Wisnewski SR, et al. Prolonged deep hypothermic circulatory arrest in rats can be achieved without cognitive deficits. Life Sci. 2007;81:543-52.

22. Rodrigo R, Fernández-Gajardo R, Gutiérrez R, Matamala JM, Carrasco R, Miranda-Merchak A, et al. Oxidative stress and pathophysiology of ischemic stroke: novel therapeutic opportunities. CNS Neurol Disord Drug Targets. 2013;12:698-714.

23. Chi W, Meng F, Li Y, Wang Q, Wang G, Han S, et al. Downregulation of miRNA134 protects neural cells against ischemic injury in N2A cells and mouse brain with ischemic stroke by targeting HSPA12B. Neuroscience. 2014;277:111-22.

24. Wang P, Liang J, Li Y, Li J, Yang X, Zhang X, et al. Down-regulation of miRNA30a alleviates cerebral ischemic injury through enhancing beclin 1-mediated autophagy. Neurochem Res. 2014;39:1279-91.

25. Irmady K, Jackman KA, Padow VA, Shahani N, Martin LA, Cerchietti L, et al Mir-592 regulates the induction and cell death-promoting activity of p75NTR in neuronal ischemic injury. $J$ Neurosci. 2014;34:3419-28.

26. Ouyang YB, Xu L, Lu Y, Sun X, Yue S, Xiong XX, et al. Astrocyte-enriched miR-29a targets PUMA and reduces neuronal vulnerability to forebrain ischemia. Glia. 2013;61:1784-94.

27. St-Pierre J, Drori S, Uldry M, Silvaggi JM, Rhee J, Jager S, et al. Suppression of reactive oxygen species and neurodegeneration by the PGC-1 transcriptional coactivators. Cell. 2006;127:397-408.

28. Arany Z, Foo SY, Ma Y, Ruas J, Bommi-Reddy A, Girnun GD, et al. HIF-independent regulation of VEGF and angiogenesis by the transcriptional coactivator PGC-1 $\alpha$. Nature. 2008;451:1008-12.

29. Gutsaeva DR, Carraway MS, Suliman HB, Demchenko IT, Shitara H Yonekawa H, et al. Transient hypoxia stimulates mitochondrial biogenesis in brain subcortex by a neuronal nitric oxide synthase-dependent mechanism. $J$ Neurosci. 2008;28:2015-24.

30. Chen SD, Lin TK, Yang DI, Lee SY, Shaw FZ, Liou CW, et al. Protective effects of peroxisome proliferator-activated receptors gamma coactivator-1alpha against neuronal cell death in the hippocampal CA1 subfield after transient global ischemia. J Biol Chem. 2007;282:2355-62.

31. Zhu HR, Wang ZY, Zhu XL, Wu XX, Li EG, Xu Y. Icariin protects against brain injury by enhancing SIRT1-dependent PGC-1alpha expression in experimental stroke. Neuropharmacology. 2010;59:70-6.

32. Fu B, Zhang J, Zhang X, Zhang C, Li Y, Zhang Y, et al. Alpha-lipoic acid upregulates SIRT1-dependent PGC-1 $\alpha$ expression and protects mouse brain against focal ischemia. Neuroscience. 2014;281:251-7.

Key Words: hypothermic circulatory arrest, brain, microRNA

\section{Discussion}

Dr J. Lawton (St Louis, Mo). You showed upregulation or more increased expression of PGC-1 alpha. Are there further experiments you could do to directly correlate that increased expression with the benefits you see? In other words, it would be difficult to inhibit it because it is so important in the mitochondrial oxidative stress.

Dr Gu. As we know, the gene transfer to the brain tissue may be more challenging than other organs because it is difficult for gene transfer vectors to get through the blood-brain barrier. That is why we chose the intracerebroventricular injection of the anti-miR-29c. Further 
experiments would be performed to explore the mechanisms of the observed cerebral protections.

Dr Lawton. But are there other ways you could specifically implicate that as the mechanism for the benefit you see? Maybe in future studies. I have a couple concerns regarding translation in the future to human benefit. So the first would be the fact that you gave your anti-miRNA 5 days before the deep hypothermic circulatory arrest. The second one is in your neurologic function you showed that there were no differences after 9 days or 14 days. In other words, the benefit of the inhibition of the miRNA was lost. Do you think these benefits would be more long term if you looked at something else?

Dr Gu. It does need some time to achieve better transfection effects after injection of lentiviral vectors. In general, what occurs more often is transient neurologic dysfunction after DHCA, such as cognitive dysfunction. We used a rat model to mimic the neurologic dysfunction after DHCA in the current study, and our results showed that anti-miR$29 \mathrm{c}$ pretreatment attenuated the transient neurologic dysfunction. There is still a long way before anti-miRNA treatment can be used clinically.

Dr Lawton. Do you have any hypotheses about why other groups have shown that miRNA is beneficial to the brain, whereas you showed that blocking the miRNA was beneficial?

Dr Gu. miRNAs negatively regulate the expressions of the target proteins. Whether the miRNA or blocking the miRNA is beneficial to the brain is decided by the functions of their target proteins.

\title{
EDITORIAL COMMENTARY
}

\section{Cerebral protection during deep hypothermic circulatory arrest: Can a molecular approach via microRNA inhibition improve on a millennia-old strategy?}

\author{
John J. Squiers, BSE, ${ }^{a}$ Mani Arsalan, MD, ${ }^{\text {,cc }}$ Brian Lima, MD, ${ }^{\mathrm{d}}$ and J. Michael DiMaio, MD ${ }^{\mathrm{a}, \mathrm{b}}$
}

See related article on pages $675-84$.

In this issue of the Journal, Wang and colleagues ${ }^{1}$ report on their investigation into cerebral protection during deep hypothermic circulatory arrest (DHCA) with a novel microRNA (miRNA) mechanism. The goal of DHCA is to cool the patient's body, effectively reducing the global cerebral metabolic rate of glucose and oxygen consumption. By decreasing the brain's metabolism, DHCA prevents cellular damage by two interrelated mechanisms: (1) it

From the ${ }^{\mathrm{a}}$ Department of Epidemiology, Baylor Healthcare System, Dallas, Tex; ${ }^{\mathrm{b}}$ The Heart Hospital Baylor Plano, Baylor Healthcare System, Plano, Tex; the ${ }^{\mathrm{c} D e p a r t-}$ ment of Cardiac Surgery, Kerckhoff Clinic Heart Center, Bad Nauheim, Germany; and the ${ }^{\mathrm{d}}$ Department of Cardiac and Thoracic Surgery, Baylor University Medical Center, Baylor Healthcare System, Dallas, Tex.

Disclosures: Authors have nothing to disclose with regard to commercial support.

Received for publication May 4, 2015; accepted for publication May 5, 2015; available ahead of print June 9, 2015.

Address for reprints: J. Michael DiMaio, MD, Baylor Healthcare System, 6125 Luther Ln, Dallas, TX 75225 (E-mail: jmdimaio@yahoo.com).

J Thorac Cardiovasc Surg 2015;150:684-6

0022-5223/ $\$ 36.00$

Copyright (C) 2015 by The American Association for Thoracic Surgery

http://dx.doi.org/10.1016/j.jtcvs.2015.05.022 lowers the demand for glucose to prevent adenosine triphosphate (ATP) depletion with concurrent anaerobic waste product accumulation that leads to necrosis, and (2) it reduces the effects of hypoxia, including activation of intracellular proteases and mitochondrial dysfunction that lead to generation of free radicals and apoptosis. ${ }^{2}$ The cerebral protection offered by DHCA is essential for the success of aortic arch surgery and complex pediatric and adult congenital cardiac procedures.

Hypothermia slows cellular metabolism by approximately $7 \%$ for every $1{ }^{\circ} \mathrm{C}$ by which the body is cooled. ${ }^{3}$ The resulting protective effect of hypothermia has been known since antiquity; Hippocrates often advocated packing the wounds of soldiers with snow and ice. ${ }^{4}$ Hypothermia was introduced into surgical practice in the mid-20th century, ${ }^{2}$ and the earliest successful open heart surgeries were implemented under hypothermic conditions. ${ }^{5}$ After Griepp and coworkers ${ }^{6}$ reported the initial experience of using hypothermia for cerebral protection during aortic arch repair, this technique was implemented worldwide in aortic surgery.

Despite its protective effects, hypothermia remains a macro level approach to limit neurologic damage during aortic surgery. Many adjunctive therapies have been 
TABLE E1. Cognitive function assessed by modified hole board test

\begin{tabular}{|c|c|c|c|c|c|c|c|c|c|}
\hline & \multicolumn{7}{|c|}{ Level of impairment } & \multirow[b]{2}{*}{ Score } & \multirow[b]{2}{*}{ Normal } \\
\hline & $\mathbf{0}$ & 1 & 2 & 3 & 4 & 5 & 6 & & \\
\hline \multicolumn{10}{|l|}{ Declarative memory } \\
\hline Wrong choice of 3 trials & 0 & 1 & 2 & 3 & 4 & 5 & $>5$ & $0-6$ & $0-1$ \\
\hline Omission errors of 3 trials & 0 & 1 & 2 & 3 & 4 & 5 & $>5$ & $0-6$ & 0 \\
\hline \multicolumn{10}{|l|}{ Working memory } \\
\hline Repeated choice of 3 trials & 0 & 1 & 2 & 3 & 4 & 5 & $>5$ & $0-6$ & $0-1$ \\
\hline \multicolumn{10}{|l|}{ Cognitive performance } \\
\hline $\begin{array}{l}\text { Time needed to complete } 3 \\
\text { trials }\end{array}$ & $<200 \mathrm{~s}$ & $200-399 \mathrm{~s}$ & $400-599 \mathrm{~s}$ & $600-799 \mathrm{~s}$ & $800-999 \mathrm{~s}$ & $1000-1199 \mathrm{~s}$ & $>2000 \mathrm{~s}$ & $0-6$ & $0-1$ \\
\hline
\end{tabular}

